\title{
Prognostic significance of non-chest pain symp- toms in patients with non-ST-segment elevation myocardial infarction
}

\author{
Inna Kim ${ }^{1}$, Min Chul Kim ${ }^{1}$, Keun Ho Park ${ }^{2}$, Doo Sun Sim ${ }^{1}$, Young Joon Hong ${ }^{1}$, Ju Han Kim \\ Myung Ho Jeong ${ }^{1}$, Jeong Gwan Cho ${ }^{1}$, Jong Chun Park ${ }^{1}$, Myeong Chan Cho ${ }^{3}$, Jong Jin Kim ${ }^{4}$, \\ Young Jo Kim ${ }^{5}$, Youngkeun $\mathrm{Ahn}^{1}$, and for the Korea Acute Myocardial Infarction Registry Investigators
}

\begin{abstract}
${ }^{1}$ Department of Cardiology, Cardiovascular Center, Chonnam National University Hospital, Gwangju; ${ }^{2}$ Department of Cardiology, Chosun University Hospital, Gwangju; ${ }^{3}$ Department of Cardiology, Chungbuk National University Hospital, Cheongju; ${ }^{4}$ Department of Cardiology, Kyung Hee University Hospital, Seoul; ${ }^{5}$ Department of Cardiology, Yeungnam University Medical Center, Daegu, Korea
\end{abstract}

Received: February 18, 2017 Revised : March 19, 2017 Accepted: March 27, 2017

\section{Correspondence to}

Youngkeun Ahn, M.D.

Department of Cardiology, Cardiovascular Center, Chonnam National University Hospital, 42 Jebongro, Dong-gu, Gwangju 61469 , Korea

Tel: $+82-62-220-4764$ Fax: +82-62-224-4764 E-mail: cecilyk@hanmail.net
Background/Aims: Chest pain is an essential symptom in the diagnosis of acute coronary syndrome (ACS). One-third of patients with ACS present atypically, which can influence their receiving timely lifesaving therapy.

Methods: A total of 617 NSTEMI patients from the Korea Acute MI Registry (KAMIR) and the Korea Working Group on MI (KorMI) databases were analyzed. The study population was divided into two groups by symptoms at presentation (typical symptoms group, 128; atypical symptoms groups, 128).

Results: In this study population, $23 \%$ of patients presented without chest pain. After propensity score matching, the contact-to-device time $(2,618 \pm 381$ minutes vs. 1,739 \pm 241 minutes, $p=0.050)$, the symptoms-to-balloon time (3,426 \pm 389 minutes vs. $2,366 \pm$ 255 minutes, $p=0.024)$, and the door-to-balloon time $(2,339 \pm 380$ minutes vs. $1,544 \pm 244$ minutes, $p=0.002$ ) were significantly higher in the patients with atypical symptoms than in those with typical symptoms, respectively. Atypical symptoms were an independent predictor for 1-year mortality (hazard ratio, 2.820; 95\% confidence interval, 1.058 to $7.515 ; p=0.038)$. The Kaplan-Meier estimates showed higher risk for 12-month mortality in patients with atypical symptoms $(p=0.048)$ and no significant difference for 12-month major adverse cardiac events $(p=0.487)$.

Conclusions: Acute myocardial infarction patients with atypical symptoms were not rare in clinical practice and showed a high risk of delayed reperfusion therapy. After imbalance between the groups was minimized by use of propensity score matching, patients who presented atypically had a high mortality rate.

Keywords: Non-ST elevated myocardial infarction; Chest pain; Propensity score

\section{INTRODUCTION}

Chest pain and discomfort are essential symptoms in the diagnosis of acute coronary syndrome (ACS). Early detection of the signs and symptoms of ACS may help in the identification of acute myocardial infarction (MI) and is crucial for optimal medical care, timely reperfu- sion therapy, and prevention of cardiac events. However, $33 \%$ of patients with ACS present without chest pain or discomfort, which can influence their receipt of timely lifesaving therapy [1].

Atypical symptoms are defined as the absence of chest pain before or during admission and can include gastrointestinal or respiratory symptoms such as dyspnea, 
nausea, vomiting, and abdominal discomfort. According to recent recommendations in the European guidelines [2], an immediate invasive strategy should be performed in the case of very-high-risk criteria in patients with nonST-segment elevation MI (NSTEMI). However, an atypical presentation of symptoms in patients with NSTEMI can delay timely reperfusion and thrombolytic therapy. In a study based on the National Registry of MI 2 (NRMI2), the absence of chest pain and the absence of discomfort at hospital presentation were the most significant factors predicting a lower use of reperfusion therapy $[3,4]$.

Whether traditional risk factors such as age, gender, and diabetes can affect the presentation of symptoms in NSTEMI patients is not well known. Only a few studies have highlighted this under-investigated subject. In comprehensive studies, atypical symptoms were noted more commonly in patients with diabetes, women, and elderly patients with ACS $[1,5]$. In a large prospective observational study, Culic et al. [5] reported that diabetes was an independent predictor of an atypical presentation of acute $\mathrm{MI}$ in men and women.

However, the data from two registries showed no differences in the incidence of pre-infarction angina between diabetic and nondiabetic groups. In that analysis, patients with diabetes were older and were more often female than those without diabetes [6]. Therefore, selection bias resulting from traditional risk factors may have been present. To address this issue, in this study we sought to use propensity score analysis to investigate the association between symptom presentation and 12-month mortality in patients with NSTEMI.

\section{METHODS}

\section{Data source}

This study was conducted by using data from the Korea Acute MI Registry (KAMIR) and the Korea Working Group on Myocardial Infarction (KorMI) databases. KAMIR is a prospective, open, observational, multicenter online registry of acute MI cases supported by the Korean Society of Cardiology. KorMI is an ongoing registry that began as a subsequent study to KAMIR. A trained study coordinator collected data by using a standardized protocol and the ethics committee of each participating hospital approved the study protocol. The Institutional
Review Board numbers of the KAMIR and KorMI studies were CNUH-2011-172 and I-2008-01-009. The study protocol conformed to the ethical guidelines of the 1975 Declaration of Helsinki, as reflected in the study approval by the human research committee of each participating institution. Written informed consent for use of the data was also obtained from each patient.

We enrolled 617 NSTEMI patients who underwent percutaneous coronary intervention (PCI) between 2008 and 2012. The study population was divided into two groups according to symptoms at hospital presentation: 474 NSTEMI patients with typical chest pain and 143 NSTEMI patients with atypical symptoms (without chest pain) were analyzed.

Typical presentation was defined as cases in which the reported symptoms included chest pain. Chest pain was defined as any symptoms of chest discomfort, sensation, or pressure or arm, neck, or jaw pain before or during admission.

Atypical symptoms were defined as the absence of chest pain before or during admission and may have included gastrointestinal or respiratory symptoms such as dyspnea, nausea, vomiting, and abdominal discomfort.

\section{Study end points}

The primary end points were 12-month mortality and major adverse cardiac events (MACEs) including allcause death, recurrent MI, cardiac death, coronary artery bypass graft surgery, and repeated PCI in NSTEMI patients during follow-up. The secondary endpoints were the contact-to-device time (defined as the time interval from first medical contact to device activation during PCI), the symptoms-to-balloon time (defined as the time interval from the patient first experiencing symptoms to balloon inflation during PCI), and the door-to-balloon time (defined as the time interval from first arrival at the hospital to balloon inflation during PCI) in the atypical and typical symptoms groups. First medical contact was defined as the first face-to-face contact made by paramedics with the patient.

\section{Statistical analysis}

Because this was a retrospective study and not a randomized controlled trial, it was necessary to reach comparability between the two groups with regard to potential confounding variables. The propensity score 
matching method has been used to minimize the bias due to confounding that frequently occurs in retrospective studies. We performed propensity score matching using one-to-one matching for atypical symptoms at hospital presentation by use of $\mathrm{R}$ software version 2.15 ( $\mathrm{R}$ Foundation). Covariates included in the model were age, body mass index, Killip class, coronary risk factors (diabetes mellitus, hypertension, hyperlipidemia, and cigarette smoking), and creatinine clearance.

Comparability of the two groups with respect to the potential confounders before (typical symptoms group, 474; atypical symptoms groups, 143) and after (typical symptoms groups, 128; atypical symptoms groups, 128) matching was also evaluated by using IBM SPSS version 22.0 (IBM Corp., Armonk, NY, USA). Continuous variables were presented as the mean value \pm standard deviation (SD) and differences were investigated by use of one-way analysis of variance (ANOVA). Discrete variables were presented as percentages and relative frequencies and comparisons were made by using chi-square tests. A logistic regression model was used to determine independent predictors of 1-year mortality and all variables considered relevant to predicting 1-year mortality were tested.

\section{RESULTS}

\section{Baseline characteristics}

The baseline characteristics of the study population before propensity score matching are summarized in Table 1. The proportion of NSTEMI patients who presented with non-chest pain complaints was $23 \%$. Patients with atypical symptom presentation were significantly older ( $74.0 \pm 9.1$ years vs. $66.4 \pm 10.6$ years, $p<0.001)$, more likely to be female (44.8\% vs. $29.1 \%, p<0.001$ ), and more likely to be hypertensive (69.2\% vs. $56.6 \%, p=0.019$ ) than patients presenting with typical symptoms, respectively. They had higher levels of serum high-sensitivity C-reactive protein (hs-CRP; $3.0 \pm 5.0 \mathrm{mg} / \mathrm{dL}$ vs. $1.6 \pm 2.9 \mathrm{mg} / \mathrm{dL}$, $p=0.005)$, serum creatinine $(1.5 \pm 1.6 \mathrm{mg} / \mathrm{dL}$ vs. $1.1 \pm 1.1$ $\mathrm{mg} / \mathrm{dL}, p=0.041)$, and serum glucose $(207.7 \pm 118.3 \mathrm{mg} / \mathrm{dL}$ vs. $117.7 \pm 79.7 \mathrm{mg} / \mathrm{dL}, p=0.011)$ and a lower left ventricular ejection fraction $(47.7 \% \pm 13.5 \%$ vs. $57.7 \% \pm 11.3 \%, p<$ 0.001 ) than did patients presenting with typical symptoms, respectively. After propensity score matching, the two-patient groups were similar in terms of all baseline covariates used for propensity score estimation (Table 2). Matching reduced covariate imbalance and improved

Table 1. Baseline clinical characteristics of NSTEMI patients before propensity score matching

\begin{tabular}{|c|c|c|c|}
\hline Characteristic & $\begin{array}{l}\text { Patient with typical symptoms } \\
\qquad(\mathrm{n}=474)\end{array}$ & $\begin{array}{l}\text { Patient with atypical symptoms } \\
\qquad(\mathrm{n}=143)\end{array}$ & $p$ value \\
\hline Age, yr & $66.4 \pm 10.6$ & $74 \pm 9.1$ & $<0.001$ \\
\hline Hypertension & $276(56.6)$ & $99(69.2)$ & 0.019 \\
\hline Smoking & $172(36.3)$ & $33(23.1)$ & $<0.001$ \\
\hline Female sex & $138(29.1)$ & $64(44.8)$ & $<0.001$ \\
\hline Diabetes mellitus & $178(37.6)$ & $64(44.8)$ & 0.143 \\
\hline Body mass index, $\mathrm{kg} / \mathrm{m}^{2}$ & $24.4 \pm 2.9$ & $23.7 \pm 2.9$ & 0.022 \\
\hline Left ventricle ejection fraction, \% & $57 \cdot 7 \pm 11.3$ & $47 \cdot 7 \pm 13 \cdot 5$ & $<0.001$ \\
\hline Plasma glucose, mg/dL & $117.7 \pm 79.7$ & $207.7 \pm 118.3$ & 0.011 \\
\hline Creatinine, mg/dL & $1.1 \pm 1.1$ & $1.5 \pm 1.6$ & 0.041 \\
\hline Total cholesterol, mg/dL & $194.2 \pm 48.2$ & $178.3 \pm 45.7$ & 0.019 \\
\hline $\mathrm{HDL}-\mathrm{C}, \mathrm{mg} / \mathrm{dL}$ & $41.6 \pm 11.4$ & $41.2 \pm 11.9$ & 0.814 \\
\hline Triglyceride, mg/dL & $133.7 \pm 76.2$ & $111.3 \pm 71.0$ & 0.025 \\
\hline LDL-C, mg/dL & $127.1 \pm 42.0$ & $116.3 \pm 40.6$ & 0.045 \\
\hline hs-CRP, mg/dL & $1.6 \pm 2.9$ & $3.0 \pm 5.0$ & 0.005 \\
\hline Pre TIMI grade flow o-2 & $327(73.2)$ & $104(69.0)$ & 0.040 \\
\hline
\end{tabular}

Values are presented as mean $\pm \mathrm{SD}$ or number (\%).

NSTEMI, non-ST-segment elevation myocardial infarction; HDL-C, high density lipoprotein cholesterol; LDL-C, low density lipoprotein cholesterol; hs-CRP, high-sensitivity C-reactive protein; TIMI, thrombolysis in myocardial infarction. 
covariate balance between the two groups. There were no statistically significant differences in any of the baseline characteristics between the groups.

After propensity score matching, the total length of hospital stay did not differ significantly between the patients with atypical symptoms and those with typical symptoms (11.7 \pm 8.2 days vs. 10.5 \pm 9.1 days, respectively; $p=0.300$ ). However, the length of stay in the coronary care unit was longer in patients with atypical symptoms $(3.7 \pm 3.4$ days vs. $3.3 \pm 2.8$ days, $p=0.047$ ) (Table 3$)$. We also analyzed the time intervals from symptom presentation and first medical contact to balloon time. As presented in Fig. 1 , the contact-to-device time $(2,618 \pm 381$ minutes vs. $1,739 \pm 241$ minutes, $p=0.050)$, the symptoms-to-balloon time $(3,426 \pm 389$ minutes vs. $2,366 \pm 255$ minutes, $p$
$=0.024)$, and the door-to-balloon time $(2,339 \pm 380 \mathrm{~min}$ utes vs. 1,544 \pm 244 minutes, $p=0.002$ ) were significantly longer in the patients with atypical symptoms than in those with typical symptoms, respectively. A hs-CRP was associated with atypical symptoms in the propensity-matched cohort (Table 4).

\section{Clinical outcomes during follow-up}

After propensity score matching, 12-month mortality was higher in the patients with atypical symptoms than in those with typical symptoms $(14.8 \%$ vs. $7.0 \%$, respectively; $p=0.045$ ) (Table 5). In the logistic regression analysis after adjustment for other variables, presentation with atypical symptoms was an independent predictor of 1-year mortality (hazard ratio [HR], 2.820; 95\% confi-

Table 2. Baseline clinical characteristics of NSTEMI patients after propensity score matching

\begin{tabular}{|c|c|c|c|}
\hline Characteristic & $\begin{array}{l}\text { Patient with typical symptoms } \\
\qquad(\mathrm{n}=128)\end{array}$ & $\begin{array}{l}\text { Patient with atypical symptoms } \\
\qquad(\mathrm{n}=128)\end{array}$ & $p$ value \\
\hline Age, yr & $74.5 \pm 8.1$ & $73.7 \pm 9.1$ & 0.320 \\
\hline Hypertension & $92(71.9)$ & $88(68.8)$ & 0.584 \\
\hline Smoking & $35(27 \cdot 3)$ & $30(23.4)$ & 0.716 \\
\hline Female sex & $50(39.1)$ & $55(43.0)$ & 0.525 \\
\hline Diabetes mellitus & $57(44 \cdot 5)$ & $58(45 \cdot 3)$ & 0.900 \\
\hline Body mass index, $\mathrm{kg} / \mathrm{m}^{2}$ & $23.8 \pm 0.12$ & $23.6 \pm 3.2$ & 0.097 \\
\hline Left ventricle ejection fraction, $\%$ & $54.6 \pm 12.7$ & $48.6 \pm 14.1$ & 0.170 \\
\hline Plasma glucose, mg/dL & $180 \pm 100$ & $183 \pm 114$ & 0.821 \\
\hline Creatinine, mg/dL & $1.5 \pm 1.8$ & $1.5 \pm 1.6$ & 0.480 \\
\hline Total cholesterol, mg/dL & $183.0 \pm 49.6$ & $175 \cdot 0 \pm 47.0$ & 0.415 \\
\hline HDL-C, mg/dL & $40.1 \pm 9.1$ & $39 \cdot 5 \pm 11.2$ & 0.605 \\
\hline Triglyceride, mg/dL & $125.2 \pm 73.5$ & $114.8 \pm 70.5$ & 0.503 \\
\hline LDL-C, mg/dL & $119.4 \pm 42.1$ & $114.8 \pm 41.8$ & 0.516 \\
\hline hs-CRP, mg/dL & $1.9 \pm 3.3$ & $2.4 \pm 4.4$ & 0.391 \\
\hline Pre TIMI grade flow o-2 & $41(32.0)$ & $31(24.4)$ & 0.400 \\
\hline
\end{tabular}

Values are presented as mean \pm SD or number $(\%)$.

NSTEMI, non-ST-segment elevation myocardial infarction; HDL-C, high density lipoprotein cholesterol; LDL-C, low density lipoprotein cholesterol; hs-CRP, high-sensitivity C-reactive protein; TIMI, thrombolysis in myocardial infarction.

Table 3. Length of hospital stay in the propensity matched cohort.

\begin{tabular}{lccc}
\hline Variable & $\begin{array}{c}\text { Patient with typical symptoms } \\
(\mathrm{n}=128)\end{array}$ & $\begin{array}{c}\text { Patient with atypical symptoms } \\
(\mathrm{n}=128)\end{array}$ & $p$ value \\
\hline Coronary care unit stay, day & $3.3 \pm 2.8$ & $3.7 \pm 3.4$ & 0.047 \\
Total hospital stay, day & $10.5 \pm 9.1$ & $11.7 \pm 8.2$ & 0.300 \\
\hline
\end{tabular}

Values are presented as mean $\pm \mathrm{SD}$. 
dence interval [CI], 1.058 to $7.515 ; p=0.038$ ). Age, hypertension, diabetes, smoking, and hs-CRP were not associated with 1-year mortality in the logistic regression analysis (Table 6).

The Kaplan-Meier estimates showed higher risk for 12-month mortality in patients with atypical symptoms $(p=0.048)$ but no difference for 12-month MACEs $(p=$ 0.487 ) (Figs. 2 and 3).

\section{DISCUSSION}

We analyzed the impact of presentation with atypical symptoms on timely revascularization and clinical outcomes in NSTEMI patients undergoing primary PCI. Before matching, $23 \%$ of the study population presented with non-chest pain complaints (44.8\% female). Previous studies have reported associations between atypical symptoms and advancing age, female gender, history of diabetes mellitus, chronic renal disease, and dementia. Therefore, such patients are at increased risk for delays in proper treatment and in-hospital mortality $[1,7,8]$. Canto et al. [9] analyzed 1.1 million patients enrolled in the National Registry of Myocardial Infarction between 1994 and 2004 and investigated sex differences in MI symptom presentation and subsequent hospital mortality after adjustment for age. However, other studies reported no statistically significant differences in the frequency of typical symptoms among women and men $[10,11]$. Several studies have reported that patients with diabetes experience less pain than do patients without diabetes, whereas other studies found no difference [8,12-15]. A review of other observational studies showed inconclusive results in terms of the association of tradi- tional risk factors such as older age, female gender, race, and diabetes with atypical symptoms. Such inconclusive

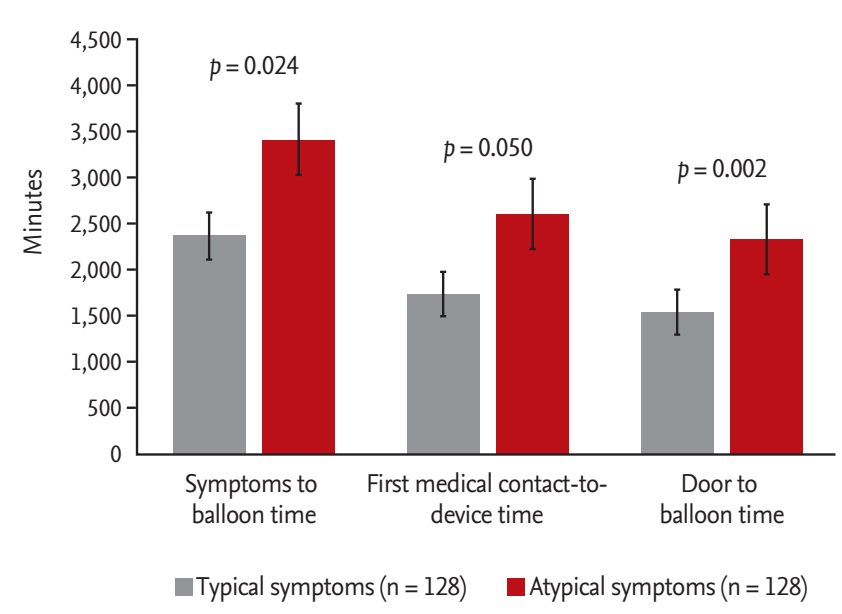

Figure 1. Time intervals according to symptoms at presentation in patients with non-ST-segment elevation myocardial infarction.

Table 4. Factors predictive of atypical symptoms in the propensity-matched NSTEMI patients

\begin{tabular}{lcc}
\hline Variable & Adjusted HR $(95 \% \mathrm{CI})$ & p value \\
\hline Age & $0.976(0.942-1.011)$ & 0.179 \\
Female & $1.472(0.719-3.011)$ & 0.290 \\
Diabetes mellitus & $0.972(0.548-1.724)$ & 0.922 \\
Hypertension & $0.717(0.372-1.381)$ & 0.320 \\
Smoking & $0.846(0.380-1.881)$ & 0.681 \\
hs-CRP & $1.086(1.006-1.173)$ & 0.035 \\
Serum creatinine & $0.922(0.771-1.103)$ & 0.375 \\
\hline
\end{tabular}

NSTEMI, non-ST-segment elevation myocardial infarction; HR, hazard ratio; CI, confidence interval; hs-CRP, highsensitivity C-reactive protein.

Table 5. Clinical outcomes of NSTEMI patients with atypical and typical symptoms in the propensity-matched cohort

\begin{tabular}{lccc}
\hline Clinical outcome & Patient with typical symptoms $(\mathrm{n}=128)$ & Patient with atypical symptoms $(\mathrm{n}=128)$ & $p$ value \\
\hline Composite of MACE & $26(20.3)$ & $17(13.3)$ & 0.132 \\
Cardiac death & $12(9.4)$ & $7(5.5)$ & 0.233 \\
All-cause death & $19(14.8)$ & $9(7.0)$ & 0.045 \\
Recurrent myocardial infarction & $4(3.1)$ & $3(2.3)$ & 0.702 \\
Any revascularization & $16(12.5)$ & $16(12.5)$ & 1.000 \\
Target lesion revascularization & $9(7.0)$ & $10(7.8)$ & 0.812 \\
\hline
\end{tabular}

Values are presented as number (\%).

NSTEMI, non-ST-segment elevation myocardial infarction; MACE, major adverse cardiac event. 
results might be explained by the heterogeneity of the population. Therefore, to reduce the effect of confounding factors on atypical complaints in the present study, we performed propensity score matching.

In the propensity-matched cohort, atypical symptoms independently predicted 1-year mortality in patients with NSTEMI (HR, 2.820; 95\% CI, 1.058 to 7.515; $p$ $=0.038$ ). However, age, diabetes, hypertension, hs-CRP, and smoking were not associated with 1-year mortality in the matched cohort.

The timely provision of optimal medical therapy or revascularization therapy is crucial in the clinical assessment of MI patients. Unfortunately, MI patients pre- senting without chest pain at the hospital often remain misdiagnosed. In the 2015 European Society of Cardiology guidelines for the management of ACS in patients presenting without persistent ST-segment elevation, an immediate invasive strategy ( $<2$ hours) was recommended for very high-risk NSTEMI patients, an early invasive strategy ( $<24$ hours) for high-risk patients, and a maximal delay of 72 hours for angiography in patients with at least one intermediate risk criterion, recurrent symptoms, or known ischemia on noninvasive testing [2]. Several randomized controlled trials have reported data showing that an early invasive strategy is safe and associated with a lower risk of refractory ischemia and

Table 6. Independent predictors of 1-year mortality in the propensity-matched NSTEMI patients

\begin{tabular}{lcccc}
\hline Variable & Unadjusted HR $(95 \% \mathrm{CI})$ & p value & Adjusted HR $(95 \% \mathrm{CI})$ & $p$ value \\
\hline Atypical symptom & $2.305(1.000-5.310)$ & 0.050 & $2.820(1.058-7.515)$ & 0.038 \\
Age & $1.028(0.980-1.079)$ & 0.258 & $1.036(0.980-1.096)$ & 0.214 \\
Hypertension & $3.054(0.534-5.546)$ & 0.997 & $2.054(0.834-2.546)$ & 0.997 \\
Diabetes mellitus & $2.431(1.075-5.500)$ & 0.033 & $1.446(0.570-3.669)$ & 0.437 \\
Smoking & $0.726(0.250-2.110)$ & 0.557 & $1.569(0.433-5.689)$ & 0.493 \\
hs-CRP & $1.095(1.008-1.190)$ & 0.032 & $1.088(0.982-10206)$ & 0.107 \\
\hline
\end{tabular}

NSTEMI, non-ST-segment elevation myocardial infarction; HR, hazard ratio; CI, confidence interval; hs-CRP, high-sensitivity C-reactive protein.

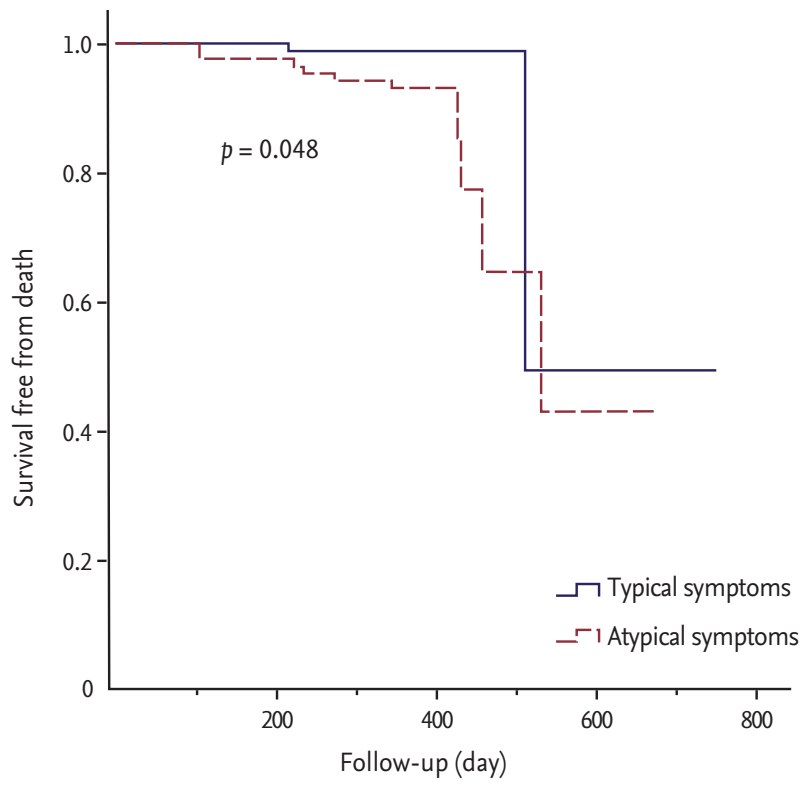

Figure 2. Kaplan-Meier curve showing all-cause death-free survival in non-ST-segment elevation myocardial infarction patients with typical and atypical symptoms.

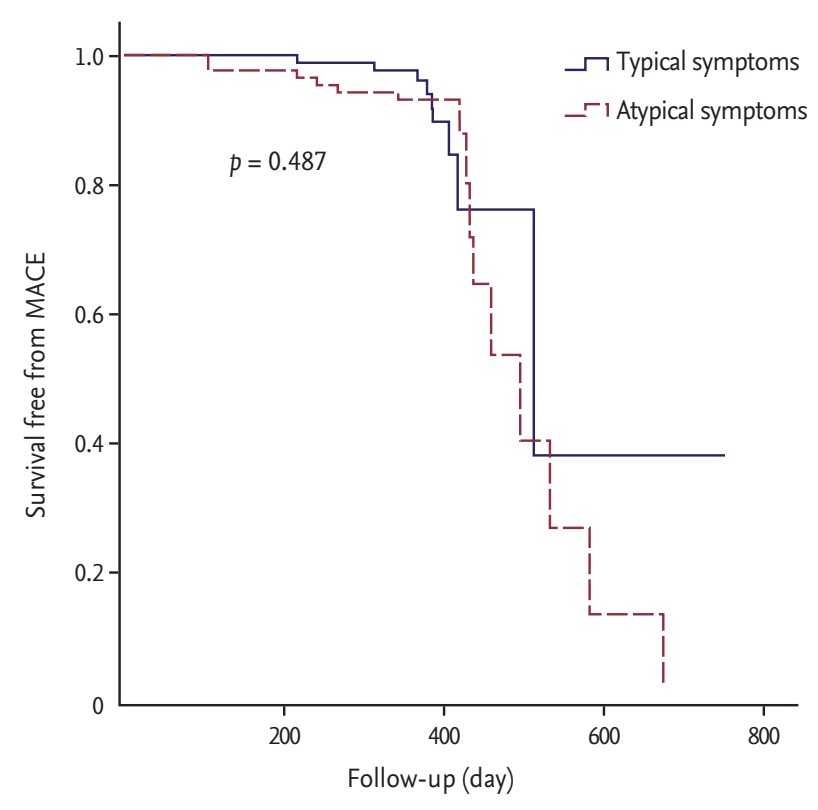

Figure 3. Kaplan-Meier curve showing MACE-free survival in non-ST-segment elevation myocardial infarction patients with typical and atypical symptoms. 
a shorter hospital stay $[16,17]$. However, atypical clinical manifestations may further delay effective reperfusion therapy. In the NRMI-2 report, patients with atypical symptom presentation had a longer delay before seeking a hospital (mean, 7.9 hours vs. 5.3 hours) [3]. Our study demonstrated that NSTEMI patients presenting with non-chest pain complaints also showed delays in the symptoms-to-balloon time, the contact-to-device time, and the door-to-balloon time. These delays may be explained by difficulties in identifying appropriate symptoms in these patients, resulting in delays in performing electrocardiograms in the emergency department.

Length of stay in the coronary care unit was longer in the group with atypical symptoms than in the typical symptoms group. This can be explained by delayed diagnosis and appropriate treatment in this group of patients.

\section{Limitations}

First, our study was not a randomized controlled trial and was based on registry data only. Second, the atypical symptoms were not well detailed and categorized except for dyspnea and our analysis was based on the presence or absence of chest pain or chest discomfort. Finally, the symptom onset time could be incorrect because it was based on the patient history.

\section{Conclusions}

In conclusion, an atypical presentation of symptoms is not rare in MI patients and can result in a delayed decision to seek medical treatment and reperfusion therapy. After we minimized the bias due to confounding between the two groups, we found that presentation with atypical symptoms independently predicted 1-year mortality in NSTEMI patients. Non-chest pain symptoms should be part of the main diagnostic criteria in MI patients. Education of not only health professionals but also the public, especially patients' family members, about the proportion of non-chest pain symptoms and their specific features can be a significant step toward prevention and prognosis of the worst clinical outcome.

\section{KEY MESSAGE}

1. The proportion of non-ST-segment elevation myocardial infarction patients who presented with non-chest pain complaints was $23 \%$.

2. After propensity score matching, contact-to-device time, symptoms-to-balloon time, and doorto-balloon time were significantly longer in patients with atypical symptoms.

3. Length of stay in the coronary care unit was longer for patients who presented with atypical symptoms.

4. Atypical symptoms independently predicted 12-month mortality (hazard ratio, 2.820; $95 \%$ confidence interval, 1.058 to $7.515 ; p=0.038$ ).

\section{Conflict of interest}

No potential conflict of interest relevant to this article was reported.

\section{Acknowledgments}

This study was supported by a grant of the National Research Foundation funded by the Korean Government (MEST), Republic of Korea (2015M3A9B4051063, 2015M3A9C6031684) and the Korean Health Technology R\&D Project, Ministry of Health and Welfare, Republic of Korea ( $\mathrm{HI}_{13} \mathrm{C}_{1527}$ ). We thank Jennifer Holmes for her English editing.

\section{REFERENCES}

1. Canto JG, Shlipak MG, Rogers WJ, et al. Prevalence, clinical characteristics, and mortality among patients with myocardial infarction presenting without chest pain. JAMA 2000;283:3223-3229.

2. Roffi M, Patrono C, Collet JP, et al. 2015 ESC guidelines for the management of acute coronary syndromes in patients presenting without persistent ST-segment elevation: task force for the management of acute coronary syndromes in patients presenting without persistent ST-segment elevation of the European Society of Cardiology (ESC). Eur Heart J 2016;37:267-315.

3. Barron HV, Rundle A, Gurwitz J, Tiefenbrunn A. Reperfusion therapy for acute myocardial infarction: observations from the National Registry of Myocardial Infarction 2. Cardiol Rev 1999;7:156-160.

4. Barron HV, Bowlby LJ, Breen T, et al. Use of reperfusion therapy for acute myocardial infarction in the United 
States: data from the National Registry of Myocardial Infarction 2. Circulation 1998;97:1150-1156.

5. Culic V, Eterovic D, Miric D, Silic N. Symptom presentation of acute myocardial infarction: influence of sex, age, and risk factors. Am Heart J 2002;144:1012-1017.

6. Kentsch M, Rodemerk U, Gitt AK, et al. Angina intensity is not different in diabetic and non-diabetic patients with acute myocardial infarction. Z Kardiol 2003;92:817-824.

7. Chen W, Woods SL, Puntillo KA. Gender differences in symptoms associated with acute myocardial infarction: a review of the research. Heart Lung 2005;34:240-247.

8. Wackers FJ, Young LH, Inzucchi SE, et al. Detection of silent myocardial ischemia in asymptomatic diabetic subjects: the DIAD study. Diabetes Care 2004;27:1954-1961.

9. Canto JG, Rogers WJ, Goldberg RJ, et al. Association of age and sex with myocardial infarction symptom presentation and in-hospital mortality. JAMA 2012;307:813-822.

10. DeVon HA, Zerwic JJ. Symptoms of acute coronary syndromes: are there gender differences? A review of the literature. Heart Lung 2002;31:235-245.

11. Mackay MH, Ratner PA, Johnson JL, Humphries KH, Buller CE. Gender differences in symptoms of myocardial ischaemia. Eur Heart J 2011;32:3107-3114.

12. Falcone C, Nespoli L, Geroldi D, et al. Silent myocardial ischemia in diabetic and nondiabetic patients with coronary artery disease. Int J Cardiol 2003;90:219-227.

13. Coronado BE, Pope JH, Griffith JL, Beshansky JR, Selker HP. Clinical features, triage, and outcome of patients presenting to the ED with suspected acute coronary syndromes but without pain: a multicenter study. Am J Emerg Med 2004;22:568-574.

14. Richman PB, Brogan GX Jr, Nashed AN, Thode HC Jr. Clinical characteristics of diabetic vs nondiabetic patients who "rule-in" for acute myocardial infarction. Acad Emerg Med 1999;6:719-723.

15. Meshack AF, Goff DC, Chan W, et al. Comparison of reported symptoms of acute myocardial infarction in Mexican Americans versus non-Hispanic whites (the Corpus Christi Heart Project). Am J Cardiol 1998;82:1329-1332.

16. Katritsis DG, Siontis GC, Kastrati A, et al. Optimal timing of coronary angiography and potential intervention in non-ST-elevation acute coronary syndromes. Eur Heart J 2011;32:32-40.

17. Navarese EP, Gurbel PA, Andreotti F, et al. Optimal timing of coronary invasive strategy in non-ST-segment elevation acute coronary syndromes: a systematic review and meta-analysis. Ann Intern Med 2013;158:261-270. 